\title{
Facteurs d'adoption de la technologie "Microdose" dans les zones agroécologiques au Burkina Faso
}

\author{
Hamadé SIGUE ${ }^{1 *}$, Innocent Adédédji LABIYI², Jacob Afouda YABI $^{2}$ et Gauthier BIAOU ${ }^{3}$ \\ ${ }^{1}$ Institut National d'Environnement et de Recherches Agricoles (INERA), \\ BP : 208, Fada N'Gourma, Burkina Faso. \\ ${ }^{2}$ Laboratoire d'Analyses et de Recherches sur les Dynamiques Economique et Sociale (LARDES), \\ Faculté d'Agronomie, Université de Parakou, BP : 123, Parakou, Bénin. \\ ${ }^{3}$ Université Nationale d'Agriculture (UNA), Porto-Novo, Bénin. \\ *Corresponding author; E-mail: hamsig65@gmail.com; Tél : +226 70247867
}

\section{REMERCIEMENTS}

L'équipe de chercheurs adresse ses remerciements au projet CRDI /ACDI N ${ }^{\circ}$ 106516-002 INuWaM "Intégration des Nutriments et de la Gestion de l'eau pour une Agriculture Durable au Sahel »financé par le Fonds Canadien de Recherche sur la Sécurité Alimentaire Internationale (FCRSAI), créé conjointement par le CRDI et l'Agence Canadienne de Développement International (ACDI).

\section{RESUME}

La disponibilité des ressources en eau et la dégradation des sols deviennent un défi majeur et menacent sérieusement les systèmes de production agricole dans la zone semi-aride du Burkina Faso. Cet article est une étude de cas, qui vise à identifier les facteurs explicatifs de l'adoption de la technologie "Microdose" avec une combinaison des techniques de gestion de l'eau et de fertilité des sols, dans les exploitations agricoles des provinces de Zondoma et de Kouritenga au Burkina Faso. La collecte des données a été effectuée auprès d'un échantillon aléatoire de 360 exploitants agricoles repartis dans les deux provinces. Dans la zone d'étude, les principales cultures vivrières sont le sorgho, le mil le maïs et le niébé qui assurent la base alimentaire des ménages agricoles. L'analyse économétrique avec le modèle logit a permis d'identifier des facteurs déterminant l'adoption du paquet technologique "Microdose". Les résultats ont montré que la formation en microdose (à $1 \%$ ), le revenu agricole (à 5\%), le nombre d'actifs (à 10\%) et l'équipement du producteur (à 10\%) ont une influence positive sur l'adoption du paquet technologique. La superficie a en revanche, une influence négative sur l'adoption au seuil de $1 \%$. Ces résultats sont susceptibles d'être exploités afin de promouvoir la production agricole dans les zones similaires.

(C) 2018 International Formulae Group. All rights reserved.

Mots clés: Fertilité des sols, microdose, conservation des sols, zone semi-aride, adoption, Burkina Faso.

\section{Adoption factors of "Microdose" technology in agro-ecological zones in Burkina Faso}

\author{
ABSTRACT \\ Water scarcity and soil quality were major factors limiting agricultural production in semi-arid zone in \\ Burkina Faso. The factors for adoption of "Microdose" technology with water and soil fertility management are
}


analyzed in this paper. The study was conducted in two provinces in Burkina Faso. Data were collected from 360 farmers chosen randomly based on focus group discussions and individual household surveys. It concerned socio-economic data and information on the use of agricultural technology in the two provinces. The logit regression model was used to determine explanatory factors for adoption. Aper-locality analysis presented variability in farmers' behaviour in the adoption of the technology package. The results show that training, formation, equipment, agricultural income and house working number have a positive influence on adoption of the package. In addition, cultivated area act negatively on adoption. These results provide insight into farmer behaviour in order to improve the production and wide dissemination of food crops such as sorghum, millet, maize and cowpea.

(C) 2018 International Formulae Group. All rights reserved.

Keywords: "Microdose" technology, water and soil conservation, semi-arid zone, adoption, Burkina Faso.

\section{INTRODUCTION}

Le problème de la dégradation de terres affecte plus de deux milliards d'hectares de terre cultivable dans le monde (El Hadraoui, 2013). La pression croissante des populations rurales sur les ressources naturelles, l'accentuation des aléas climatiques et les capacités d'investissement limitées des exploitations agricoles familiales ont aussi entraîné progressivement une dégradation des agrosystèmes tropicaux et méditerranéens (Dugué et al., 2015). En réponse à ces préoccupations, les techniques de microdose combinées avec celles de la gestion de l'eau et des sols sont présentées comme des solutions de nos jours. De plus, l'amélioration des recommandations en matière d'engrais pour un meilleur retour sur investissement dans les exploitations est une meilleure réponse optimisée aux cultures aux engrais (Bationo et al., 2018).

$\mathrm{Au}$ Burkina Faso, les provinces du Zondoma et du Kouritenga sont particulièrement affectées par les effets des changements climatiques depuis les sécheresses des années 1970. On y enregistre une diminution accélérée des ressources naturelles et le fort taux d'accroissement de la population a exacerbé une pression sur les terres cultivables (CILSS, 2010). Les principales cultures pratiquées dans ces localités sont les cultures de mil, du sorgho, du maïs et du niébé. La productivité agricole au Burkina Faso d'une manière générale est faible et les ménages agricoles vivent dans une situation de précarité alimentaire et d'une pauvreté de plus en plus accentuée (Natasha et al., 2017).

Déjà dans les années 1980, l'Etat burkinabè, la recherche agronomique et les partenaires au développement ont mis en œuvre différents projets et programmes visant le transfert de technologies agricoles. Ces actions visaient l'accroissement de la productivité des principales cultures vivrières telles que le sorgho, le mil, le maïs et le niébé du fait de leur importance pour la sécurité alimentaire et la lutte contre la pauvreté (Ouédraogo, 2010). Des technologies ont été développées pour la restauration des sols dégradés à travers la conservation des eaux et des sols (CES/DRS), la gestion intégrée de la fertilité des sols (GIFS) généralement accompagnées par l'utilisation efficiente et ciblée de l'engrais minéral à petites doses.

En 2011, le projet CRDI/ACDI $\mathrm{N}^{\circ}$ 106516-002 «Intégration des Nutriments et de la Gestion de l'Eau pour une Agriculture Durable au Sahel», afin d'améliorer et d'accroître les productions vivrières, a procédé à un processus de diffusion et d'apprentissage de différentes technologies auprès des producteurs. Les actions combinaient à la fois la pratique de la microdose avec les autres techniques de CES/DRS, de GIFS et ont amélioré les rendements des cultures vivrières. Les résultats obtenus lors des tests de démonstrations ont enregistré des performances technologiques de l'ordre de $30 \%$ voire $40 \%$ (Ouédraogo, 2012). En effet, $9 \%$ des producteurs touchés lors de la phase d'expérimentation de la technologie sont 
devenus adoptants. Mais aussi, plus de la moitié (54\%) des adoptants l'ont réalisé sur des petites superficies (moins de $1 \mathrm{ha}$ ). La question qui se pose est donc de savoir pourquoi, dans l'ensemble, la superficie d'adoption de la technologie "Microdose" est aussi faible? Quels en sont les facteurs explicatifs de l'adoption de cette technologie? L'objectif de la présente étude est d'analyser les facteurs explicatifs de l'adoption de la technologie "Microdose" dans les provinces de Zondoma et de Kourittenga au Burkina Faso.

\section{MATERIEL ET METHODES \\ Zone de l'étude}

L'étude s'est déroulée dans deux (02) provinces $\mathrm{du}$ plateau central burkinabè (Zondoma localisé dans la région du Nord et Kouritenga dans la région du Centre-Est). Ces provinces sont situées dans une zone climatique comprise entre les isohyètes 400 et $800 \mathrm{~mm} / \mathrm{an}$ et correspond à la zone d'intervention du projet «Intégration des Nutriments et de la Gestion de l'eau pour une Agriculture Durable au Sahel ». Ce sont deux localités agroécologiques nord-soudaniennes qui présentent des systèmes de production relativement homogènes avec les cultures de mil, sorgho, maïs et niébé et sont confrontées à des contraintes similaires de faible productivité agricole. Elles ont bénéficié auparavant dans les années 1980 de la diffusion de technologies agricoles CES/DRS. Récemment en 2011, ces provinces ont bénéficié de l'intervention de projets de gestion intégrée de la fertilité avec des tests de démonstration de la microdose, la gestion de l'eau et de la fertilité des sols.

$\mathrm{Au}$ niveau de chaque province, des communes qui sont des entités administratives ont été retenues suivant un gradient nord-sud. Les villages ont été retenus au niveau des communes suivant le même gradient nord sud pour tenir compte de la localisation communale et de la cartographie des variations climatiques et de la diversité agroécologique (Figure 1).

\section{Echantillonnage et collecte de données}

La base de sondage de l'enquête repose sur les producteurs de vivriers dans la zone d'intervention du projet en 2011. Dans les deux provinces, l'échantillon a concerné des producteurs le sorgho, le mil le maïs et le niébé impliqués dans le projet.

La stratification pour les besoins de l'enquête a d'abord été par commune puis les villages suivant un gradient nord-sud. L'échantillon comporte six (06) villages retenus dans la zone : 03 villages par province à raison d'un village par commune (Tableau 1). Ce sont 180 producteurs qui ont été tirés de façon aléatoire par province. Au total, 360 ménages adoptants et non adoptants de la technologie ont constitué l'échantillon et ont effectivement été enquêtés. Cet échantillonnage a pris en compte l'aspect genre, jeunes et adultes avec 30\% de femmes.

Cette collecte de données s'est déroulée en 2013 en opérant une typologie des ménages élaborée en lien avec la vulnérabilité et l'adoption des techniques de gestion de l'eau et de la fertilité des sols (technologie "Microdose"). Les données ont été collectées suivant les focus groups et l'enquête individuelle de ménage semi structurée.

L'enquête ménage a concerné les chefs de ménage adoptant le paquet technologique "Microdose" ou non. Les données collectées ont regroupé : (i) les caractéristiques socioéconomiques des ménages et démographiques; (ii) les indicateurs de la vulnérabilité ; (iii) les indicateurs de l'adoption des technologies, (iv) et la sécurité alimentaire (la disponibilité alimentaire) au niveau des ménages.

Les données ont été saisies à l'aide du logiciel Sphinx et ont été analysées avec le logiciel STATA 13.

\section{Technologie "Microdose"}

Dans le contexte de notre étude, la technologie "Microdose" est la pratique de l'une des composantes suivantes, diffusées dans la zone d'étude :

- Microdose (MD) avec Cordons pierreux (CP) avec Compost (CO) ; 
- Microdose (MD) avec Cordons pierreux (CP) avec Fumure organique (FO) ;

- Microdose (MD) avec Cordons pierreux (CP) avec Paillage (PA) ;

- Microdose (MD) avec Bandes enherbées (BE) avec Compost (CO) ;

- Microdose (MD) avec Bandes enherbées (BE) avec Fumure Organique (FO) ;

- Microdose (MD) avec Bandes enherbées (BE) avec Paillage (PA) ;

- Microdose (MD) avec Demi-lunes (DL) avec Compost (CO) ;

- Microdose (MD) avec Demi-lunes (DL) avec Fumure organique (FO) ;

- Microdose (MD) avec Zaï avec Compost (CO) ;

- Microdose (MD) avec Zaï avec Fumure organique (FO).

\section{Modèle conceptuel}

Plusieurs études empiriques ont étudié

l'adoption des innovations agricoles (Nkamleu et Coulibaly, 2000 ; Adésina et al., 2000 ; Kini, 2007 ; Koutou, 2007). La revue de la littérature sur les études d'adoption des innovations permet de distinguer trois types de modèles fréquemment utilisés pour analyser la décision d'adopter une technologie agricole : les modèles de probabilité linéaire, logit et probit. Les modèles de probabilité linéaire présentent des inconvénients car la probabilité peut souvent dépasser 1. Quant aux modèles logit et probit, ils sont souvent utilisés dans la plupart des études d'adoption. Dans cette étude, la modélisation économétrique avec un modèle logit comme outil d'analyse a été utilisée parce qu'il a été jugé appropriée dans la spécification des relations entre la décision d'adopter et les facteurs déterminants de l'adoption. On suppose, en effet, que l'adoption d'une des composantes de la technologie "Microdose" par les producteurs agricoles tirés, suit une loi logistique.

La décision d'adoption du paquet technologique "Microdose" résulte d'un calcul économique coût-bénéfice par le producteur. Avant de se décider, le producteur compare le coût d'acquisition de la technologie par rapport au gain qu'il peut réaliser en adoptant la technologie. Le coût et le gain sont des informations privées détenues par le producteur. Lorsque nous considérons une variable qui puisse prendre en compte les informations privées détenues par le producteur. Une telle variable est qualifiée de variable latente et est continue.

Soit Y la variable latente représentant la décision du producteur.

$\mathrm{Y}=\beta X_{i}+\epsilon_{i}$

Avec $\beta^{\prime}=$ le vecteur des paramètres à estimer, $X_{i}$ représente les variables explicatives, $\epsilon_{i}$ les termes d'erreurs.

On a les cas suivants :

Si $\mathrm{Y}^{*}>0$ alors le producteur adopte la technologie, donc $y_{i}=1$

$\mathrm{Si} \mathrm{Y}^{*} \leq 0$ alors le producteur n'adopte pas la technologie, $y_{i}=0$

Considérons le cas où le producteur adopte $\left(y_{i}=1\right)$. Alors la probabilité $\mathrm{p}$ est donnée par:

$p\left(y_{i}=1\right)$ signifie $p\left(\mathrm{Y}^{*}>0\right)=p\left(\beta^{\prime} X_{i}+\epsilon_{i}>0\right)$ Alors :

$p\left(\epsilon_{i}>-\beta^{\prime} X_{i}\right)=F\left(\beta^{\prime} X_{i}\right)$

On fait l'hypothèse que les erreurs $\epsilon_{i}$ sont indépendantes et identiquement distribuée et suivent la loi logistique. La modélisation logistique de $\mathrm{y}$ en fonction de $\mathrm{p}$ variables explicatives $\left(X_{1}, X_{2}, \ldots, X_{p}\right)$ et de $\mathrm{p}+1$ paramètres à estimer $\left(\beta_{0}, \beta_{1}, \beta_{2}, \ldots, \beta_{p}\right)$ est :

$p\left(y=1 \backslash X_{1}=x_{1}, X_{2}=x_{2}, \ldots, X_{p}=x_{p}\right)=$ $\frac{e^{\beta_{0}+\sum_{i=1}^{p} \beta_{i} x_{i}}}{1+e^{\beta_{0}+\sum_{i=1}^{p} \beta_{i} x_{i}}}$

De (4), la fonction de lien logistique devient :

$\operatorname{logit}\left(y=1 \backslash X_{i}=x_{i}\right)=\beta_{0}+\sum_{i=1}^{p} \beta_{i} x_{i}, i=\overline{1, n}$

En introduisant le terme d'erreur, on obtient le modèle économétrique suivant :

$\operatorname{logit}\left(y=1 \backslash X_{i}=x_{i}, i=\overline{1, n}\right)=\beta_{0}+$ $\beta_{1} x_{1}+\beta_{2} x_{2}+\cdots+\beta_{p} x_{p}+\epsilon_{i}$

\section{Spécification du modèle empirique}

Le modèle logit binaire a été utilisé pour déterminer les variables socioéconomiques ayant une influence sur l'adoption de la technologie "Microdose" (Tableau2). La situation de référence choisie est celle où la variable dépendante «adoption» prend la 
valeur un (1) si le producteur adopte la technologie Microdose, et zéro (0), sinon.

\section{Variable expliquée}

La variable expliquée est l'adoption (ADOPTION). Dans le modèle, l'adoption représentée par le code (adoption) et est supposé divisible. De plus, l'hypothèse de divisibilité de la technologie "Microdose" est faite de sorte à ce que le producteur puisse, en fonction de sa perception des composantes, faire un choix. Ainsi, il a été considéré qu'un producteur a adopté la technologie "Microdose", lorsqu'il dispose d'une superficie non nulle ayant bénéficiée d'au moins d'une composante de la technologie "Microdose".

\section{Variables explicatives}

Sexe (SEXE): Dans les études d'adoption de technologies, les questions du genre ont généralement été abordées. Une étude de Obisesan (2014) sur l'adoption de nouvelles technologies a révélé que le sexe avait une influence à la fois significative et positive sur l'adoption. Les résultats des études montrent que les femmes ont un espace d'autonomie économique (Mignouna et al., 2011 ; Salé et al., 2014 ; Yabi et al., 2016). Elles ont des champs personnels où elles pratiquent diverses cultures (Droy, 2011). Le sexe serait un facteur déterminant de l'adoption de nouvelles techniques agricoles. Son signe théorique attendu serait positif pour les hommes et négatif pour les femmes.

Age (AGE) : L'âge du chef d'exploitation agricole a une incidence sur la probabilité d'adoption de la technologie de "Microdose". Les agriculteurs plus âgés ont acquis des connaissances et une expérience au fil du temps et sont mieux en mesure d'évaluer l'information technologique que les jeunes agriculteurs (Mignouna et al, 2011 ; Kariyasa et al., 2011 ; Folefack et al., 2012). A cet effet, l'âge pourrait avoir une incidence positive sur la probabilité d'adoption. Ainsi, le signe théorique attendu est positif.

Superficie (SUPERF) :

Elle

représente la superficie cultivée d'au moins 0,25 hectare. Une plus grande superficie impose un coût d'investissement élevé pour l'acquisition de la technologie 'Microdose'. Il est démontré que la superficie cultivée est négativement corrélée à la probabilité d'adoption de la technologie de micro dose (Soulé et al., 2000 ; Anderson et al., 2005). Le signe attendu est de ce fait négatif.

Equipement (EQPMT) : C'est la disponibilité d'un matériel pour l'exécution des opérations agricoles. Les paysans investissent dans l'achat de matériel de production tels que les charrues, les charrettes et les animaux de traits (Traoré et al., 2013 ; Andrianirina, 2013) nécessaires pour la mise en œuvre des itinéraires techniques. La disponibilité en équipement constitue pour le producteur un facteur d'amélioration de la productivité agricole. Ainsi, son influence sur l'adoption de la technologie est positive.

Formation (FORM) : $\mathrm{La}$ variable formation signifie que le producteur a bénéficié d'une formation en techniques de production agricole. En théorie, la formation permet aux paysans d'avoir une connaissance de la technologie et de son utilisation. Un producteur encadré et suivi par le service de vulgarisation dispose d'une propension à adopter la technologie par rapport à un producteur qui n'a pas reçu d'encadrement (Rogers, 1983 ; Kaboré al., 2011). Comme Adekambi (2010), un effet positif de cette variable est attendu.

Nombre d'actifs: (NBACT) : il correspond à l'ensemble formé des hommes et femmes de plus 15 ans, participant effectivement aux activités agricoles au sein du ménage. Le nombre d'actifs dans l'exploitation agricole contribue à l'exécution de la technologie agricole. Comme l'atteste Pender et Ndjeunga (2008), le nombre d'actifs est source de main d'œuvre pour l'effort d'investissement dans l'adoption des technologies (Adeoti, 2002). Le signe théorique peut être positif.

Revenu agricole (REVAG) : Le revenu brut agricole représente le produit en valeur issu de la vente des produits agricoles. C'est un facteur très important dans la gestion du risque. L'exploitant agricole rationnel, cherche à maximiser sa production et en partant, son revenu agricole. Le signe théorique attendu est positif.

Revenu extra agricole (REVEX) :

Théoriquement, le revenu issu d'une activité 
indépendante, non agricole peut être utilisé pour investir dans l'adoption de technologies. Le revenu non agricole contribue à financer les dépenses de production afin de générer plus de revenu pour cette activité. Une étude menée par Pénot (2014) a montré que la principale activité en tant que travail hors exploitation (off farm) est le salaire agricole local, il représente entre 25 et $30 \%$ des activités secondaires pratiquées par les ménages dans la région du lac Alaotra au Madagascar. Dans ces conditions, le producteur est prédisposé à allouer une partie de ce revenu à l'adoption des technologies agricoles. De ce fait, la relation théorique supposée est positive.

Localité (LOC): Les conditions agroécologiques de la zone peuvent avoir une influence sur l'adoption des technologies.
Moins la zone est arrosée, plus l'adoption des technologies est élevée. Il existe d'importantes variations dans les conditions locales qui nécessitent des réponses bien adaptées. Les producteurs ont des aspirations et des préoccupations liées à leurs caractères spécifiques selon la localité. Le signe attendu est ambigüe.

Suivant les variables retenues, le modèle d'estimation (5) prend la forme suivante :

$$
\begin{aligned}
& \text { ADOPTION } \\
& =\beta_{0}+\beta_{1} \mathrm{AGE}+\beta_{2} \text { SEXE }+\beta_{3} \text { SUPERF } \\
& +\beta_{5} \text { EQPMT }+\beta_{6} \text { FORM }+\beta_{7} \text { NBACT } \\
& +\beta_{8} \text { REVAG }+\beta_{8} \text { REVEX }+\beta_{9} \text { LOC } \\
& +\epsilon \quad(6)
\end{aligned}
$$

Les caractéristiques des variables explicatives pour les analyses sont consignées dans le Tableau 2.

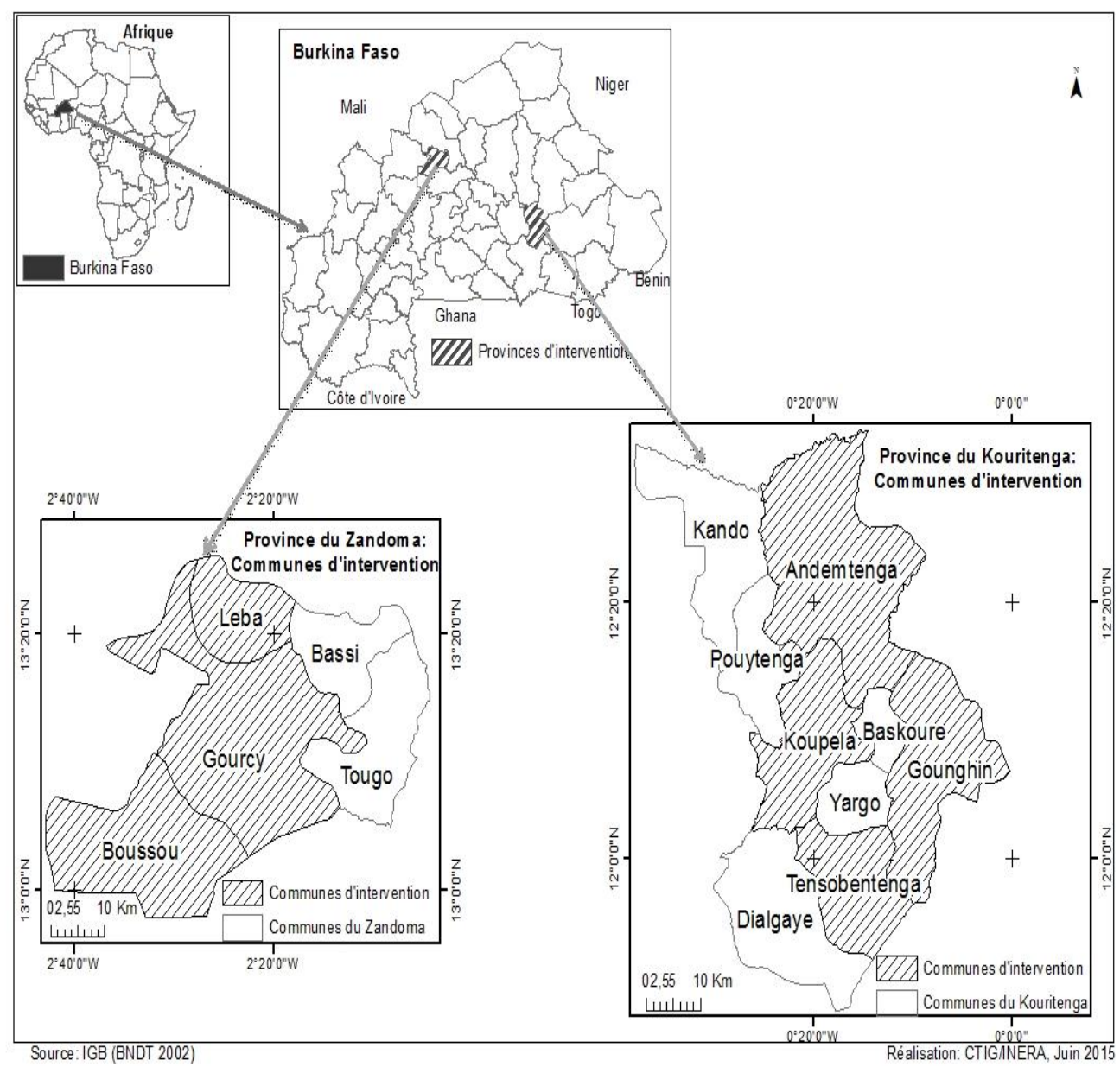

Figure 1: Sites de la zone d'étude. 
Tableau 1 : Structure de l'échantillon.

\begin{tabular}{|c|c|c|c|c|c|}
\hline Provinces & Communes & Villages & $\begin{array}{c}\text { Taille de } \\
\text { l'échantillon }\end{array}$ & Adoptants & Non adoptants \\
\hline \multirow[t]{4}{*}{ Kouritenga } & Andemtenga & Songretenga & 60 & & \\
\hline & Koupèla & Ronsin & 60 & 167 & 13 \\
\hline & Gounghin & Kabeiga & 60 & & \\
\hline & Total & & 180 & 167 & 13 \\
\hline \multirow[t]{4}{*}{ Zondoma } & Lèba & Raguèguema & 60 & & \\
\hline & Gourcy & Kagapessogo & 60 & 160 & 20 \\
\hline & Boussou & Boussou & 60 & & \\
\hline & Total & & 180 & 160 & 20 \\
\hline Zone d'étude & - & - & 360 & 327 & 33 \\
\hline
\end{tabular}

Tableau 2 : Description des variables explicatives.

\begin{tabular}{|c|c|c|c|c|}
\hline $\mathbf{N}^{\circ}$ & Nom des variables & Abréviations & Modalités & Signes attendus \\
\hline 1 & $\begin{array}{c}\text { Age } \\
\text { Moins de } 30 \text { ans } \\
\text { De } 30 \text { à } 45 \text { ans } \\
\text { De } 45 \text { à } 65 \text { ans } \\
\text { Plus de } 65 \text { ans }\end{array}$ & AGE & $\begin{array}{l}0=\text { non } ; 1=\text { oui } \\
0=\text { non } ; 1=\text { oui } \\
0=\text { non } ; 1=\text { oui } \\
0=\text { non } ; 1=\text { oui }\end{array}$ & + \\
\hline 2 & Sexe & SEXE & $0=$ féminin $; 1=$ masculin & + \\
\hline 3 & Superficie & SUPERF & - & - \\
\hline 4 & Equipement agricole & EQPMT & $0=$ non $; 1=$ oui & + \\
\hline 5 & Formation & FORM & $0=$ non $; 1=$ oui & + \\
\hline 6 & Nombre d'actifs & NBACT & - & + \\
\hline 6 & Revenu agricole & REVAG & - & + \\
\hline 7 & Revenu extra-agricole & REVEX & - & - \\
\hline 8 & $\begin{array}{c}\text { Localité } \\
\text { Kouritenga } \\
\text { Zondoma }\end{array}$ & LOC & $\begin{array}{l}0=\text { non } ; 1=\text { oui } \\
0=\text { non } ; 1=\text { oui }\end{array}$ & $+/-$ \\
\hline
\end{tabular}

\section{RESULTATS}

Caractéristiques socio-économiques et démographiques des ménages agricoles

Dans les deux provinces, la plupart des répondants sont des hommes $(74 \%)$ qui possèdent le plus souvent les terres (Tableau 3). Les exploitants enquêtés ont un âge compris entre 18 à 75 ans avec une moyenne de 45 ans. Les jeunes hommes s'intéressent plus à des activités non agricoles (petit commerce, orpaillage et autres prestations de services) et migrent vers les centres urbains laissant les activités agricoles au profit des plus âgés. La majorité des répondants $(71 \%)$ sont analphabètes. En effet, $11,7 \%$ des enquêtés ont le niveau primaire et $0,8 \%$ au secondaire. L'alphabétisation en langue et l'école coranique occupent respectivement $9,9 \%$ et $7,5 \%$ des répondants.

La tenure foncière est appréhendée par le mode d'accès à la terre. A ce sujet, $88,6 \%$ des exploitants agricoles interrogés sont 
propriétaires par héritage et les autres, principalement les allochtones, les empruntent. Les principales spéculations qui sont le sorgho mil maïs et niébé occupent une place importante dans l'alimentation et aussi dans la vie sociale des populations de cette région.

S'agissant des superficies cultivées, $65 \%$ des exploitants enquêtés ont déclaré exploiter une superficie comprise entre 0,5 à 4,8 ha. Le sorgho ou le mil sont souvent en association avec le niébé.

Le revenu agricole moyen par producteur au niveau de l'échantillon est estimé à 138635 FCFA mais présente des variations entre localité et entre les producteurs d'une même localité tandis que le revenu extra agricole moyen par producteur s'élève environ à 99250 FCFA.

Les adoptants de la technologie "Microdose" sont majoritairement en contact avec les services de vulgarisation agricole à travers les séminaires de formation organisés à leur intention. Malgré tout, le taux d'adoption de la technologie "Microdose" sur une superficie de plus de 1 ha est seulement de 36\% (Tableau 3).

\section{Déterminants de l'adoption de la technologie "Microdose" dans le Kouritenga et le Zondoma}

Le modèle estimé est globalement significatif au seuil de $1 \% \quad(\mathrm{p}=0,000)$ (Tableau 4). Ainsi, il est possible de conclure que le résultat d'estimation est efficace. L'estimation économétrique montre que les variables qui affectent significativement l'adoption de la technologie "Microdose" sont la superficie $(1 \%)$, l'équipement $(10 \%)$, la formation (1\%), le nombre d'actifs (10\%) et le revenu agricole.

En effet, la formation du producteur aux techniques de production agricole et son équipement influencent significativement l'adoption des composantes de la technologie "Microdose" et ont respectivement un effet marginal positif de 0,16 et 0,013 . Cela traduit que lorsque le producteur bénéficie d'une formation additionnelle et bien équipé, plus il songe à l'adoption de la technologie "Microdose". Ce résultat indique que la formation est un facteur qui améliore fortement probabilité d'adoption. Ainsi, les producteurs ayant bénéficié des formations en techniques agricoles sont les meilleurs adoptants. Ce résultat révèle aussi que la possession d'un équipement agricole constitue pour la production un facteur favorisant l'adoption de la technologie combien exigeante en main d'œuvre.

Le nombre d'actifs agricoles a une influence positive sur l'adoption de la technologie "Microdose" avec un effet marginal de 0,002. L'explication de ce résultat tient au fait que dans l'immense majorité des exploitations agricoles, ce sont les membres de la famille actifs qui constituent la force de travail. Ceci étant, plus la d'œuvre familiale est disponible dans la zone, plus il y a de chance que les producteurs adoptent cette technologie.

Le revenu agricole est aussi un facteur stimulant la décision d'adoption de la technologie bien que son effet marginal soit faible. Pour les producteurs, il est fort probable d'adopter la technologie "Microdose" lorsque leur revenu agricole s'améliore.

Contrairement aux variables précédentes, la superficie a un effet significatif marginal négatif $(-0,003)$. Cela signifie qu'une hausse de la superficie réduit la possibilité d'adoption de la technologie dans les deux provinces. Ce résultat montre la capacité d'absorption de la technologie en main d'œuvre et ainsi l'efficacité productive de l'exploitant. 
Tableau 3: Statistiques descriptives des caractéristiques socio-économiques et démographiques des répondants.

\begin{tabular}{|c|c|c|c|c|}
\hline Indicateurs & Modalités & Kouritenga & Zondoma & Ensemble \\
\hline \multirow[t]{2}{*}{ Genre (\%) } & Homme & 79 & 69 & 74 \\
\hline & Femme & 21 & 31 & 26 \\
\hline \multirow{4}{*}{$\begin{array}{l}\text { Catégorie } \\
\text { d'âges } \\
\text { moyens (\%) }\end{array}$} & Age1 $:<30$ ans & 2,8 & 2,8 & 2,8 \\
\hline & Age $2: 30$ à 45 ans & 16,1 & 10,9 & 13,5 \\
\hline & Age $3: 46$ à 65 ans & 24,8 & 27,3 & 26,1 \\
\hline & Age $4:>65$ ans & 1,8 & 4,9 & 3,5 \\
\hline \multicolumn{2}{|c|}{ Nombre d'actifs par ménage } & $4,48(3,10)$ & $4,18(2,81)$ & $4,33(2,96)$ \\
\hline \multirow{3}{*}{$\begin{array}{l}\text { Superficie } \\
\text { emblavée }\end{array}$} & Superficie moyenne (ha) & $5,62(0,24)$ & $4,05(0,14)$ & $4,80(0,14)$ \\
\hline & Superficie MD $(\leq 1$ ha $)(\%)$ & 56 & 51 & 54 \\
\hline & Superficie MD ( $\geq 1$ ha) $(\%)$ & 37 & 38 & 37 \\
\hline \multirow{3}{*}{$\begin{array}{l}\text { Revenu } \\
\text { moyen }\end{array}$} & Revenu agricole (FCFA) & $172515(11255,85)$ & $104755(6122,5)$ & $138635(6642,55)$ \\
\hline & Revenu non agricole (FCFA) & $112500(7198,75)$ & $85998(4989,18)$ & $99249(4428,79)$ \\
\hline & Equipement (\%) & 53 & 29 & 41 \\
\hline \multirow{3}{*}{ Adoption } & Effectif adoptants & 167 & 160 & 163 \\
\hline & Effectif adoptants formés & 119 & 87 & 206 \\
\hline & Effectif adoptants non formés & 48 & 73 & 121 \\
\hline
\end{tabular}

( ) = écart-type

Tableau 4 : Résultats de l'estimation du modèle logit.

\begin{tabular}{|c|c|c|c|}
\hline \multicolumn{2}{|c|}{ Variables } & Coefficients & Effets marginaux \\
\hline \multicolumn{2}{|c|}{ Sexe } & $0,409(0,567)$ & \\
\hline \multicolumn{2}{|c|}{ Superficie emblavée } & $-0,296(0,065) * * *$ & $-0,003$ \\
\hline \multicolumn{2}{|c|}{ Equipement } & $1,366(0,736)^{*}$ & 0,013 \\
\hline \multicolumn{2}{|c|}{ Formation } & $5,059(1,006) * * *$ & 0,165 \\
\hline \multicolumn{2}{|c|}{ Nombre d'actifs agricoles } & $0,229(0,121)^{*}$ & 0,002 \\
\hline \multirow{4}{*}{$\stackrel{8}{4}$} & Moins de 30 ans & $-0,082(0,093)$ & - \\
\hline & De 30 à 45 ans & $-0,085(0,066)$ & - \\
\hline & De 45 à 65 ans & $-0,046(0,049)$ & - \\
\hline & Plus de 65 ans & $-0,040(0,042)$ & - \\
\hline \multicolumn{2}{|c|}{ Localité (Zondoma) } & $-0,435(0,502)$ & - \\
\hline \multicolumn{2}{|c|}{ Revenu agricole } & $0,008(0,000) * *$ & $8,35 \mathrm{e}-08$ \\
\hline \multirow{2}{*}{\multicolumn{2}{|c|}{$\begin{array}{l}\text { Revenu extra agricole } \\
\text { constante }\end{array}$}} & $0,004(0,003)$ & - \\
\hline & & $3,262(2,372)$ & - \\
\hline \multicolumn{2}{|c|}{ LR chi2(12) } & & $=82,06$ \\
\hline \multicolumn{2}{|c|}{ Probabilité > chi2 } & & $=0,0000$ \\
\hline \multicolumn{2}{|c|}{ Pseudo $\mathrm{R}^{2}$} & & $=0,3720$ \\
\hline \multicolumn{2}{|c|}{ Log likelihood } & & $=-69,265554$ \\
\hline
\end{tabular}




\section{DISCUSSION}

Dans les sites d'étude, sur un total de 360 producteurs enquêtés, 327 ont déclaré avoir adopté la technologie "Microdose" soit un taux d'adoption de 90,83\%. Ce taux d'adoption est comparable à ceux obtenus par de nombreux auteurs (Gedikoglu et al., 2011 ; Mariano et al., 2012; Baffoe-Asare et al., 2013 ; Barry, 2017).

La formation en techniques agricoles est un facteur déterminant de l'adoption de la technologie 'Microdose'. Ce résultat est similaire à ceux obtenus par Sauer et Zilberman (2009), Gedikoglu et al. (2011) et Baffoe-Asare et al. (2013) pour qui, la formation constitue un facteur stimulant la décision d'adoption d'une nouvelle technologie agricole. Tout comme ces derniers, Dercon et Christiaens (2011) ont également trouvé que la connaissance et l'éducation sont de déterminants importants de l'adoption de la technologie "Microdose". En accord avec tous ces auteurs, ce résultat contribue à confirmer notre hypothèse de départ.

Des études ont montré l'effet positif de l'équipement sur l'adoption. Mariano et al. (2012) ont précisé que l'équipement contribue à renforcer la décision d'adoption de technologie. Aussi, les résultats de Blazy et al. (2011) ; Rodríguez-Entrena et Arriaza (2013) dans le cadre de l'adoption d'innovations comme les pratiques innovantes ou de conservation confirment davantage ce lien positif. Ce résultat contribue à confirmer la première hypothèse de l'analyse.

Le niveau des actifs du ménage influence l'adoption de la technologie "Microdose". Dans la littérature empirique, des auteurs comme Baffoe-Asare et al. (2013) ont mis en exergue la contribution positive du capital social sur l'adoption. Bien avant, Gedikoglu et McCann (2007) ; Gedikoglu et al. (2011) ont soutenu que le nombre d'actif génère la pluriactivité, ce qui facilite l'adoption d'innovations qui ne nécessitent pas forcément d'investissements matériels. Ce résultat contribue à confirmer la première hypothèse de l'analyse.
L'explication de ce résultat lié au revenu agricole réside dans le fait que le revenu agricole du producteur constitue une source principale pour l'investissement dans l'innovation technologique. C'est ainsi que Bradshaw (2007) ; Rodríguez-Entrena et Arriaza (2013) avaient conclu que le revenu agricole conditionne l'investissement et permet aussi de supporter des pertes à court terme lors de la mise en place de l'innovation. Cet investissement peut aussi passer par l'achat de la fumure minérale (Bello et al., 2012).

L'analyse de l'élasticité associée à la variable superficie soutient qu'une hausse la superficie de $1 \%$ entraine une baisse de la probabilité d'adoption de $0,013 \%$. En effet, lorsque la superficie consacrée à la culture agricole augmente, cela représente pour le producteur des coûts d'investissements supplémentaires pour l'acquisition de la technologie micro dose. Ce résultat est similaire à celui d'Anderson et al. (2005) qui obtiennent un effet négatif de l'accroissement de la taille de l'exploitation sur l'adoption. De même, Soule et al. (2000) ont révélé que la taille de l'exploitation implique aussi plus de contraintes financières pour l'exploitant ce que qui peut freiner l'adoption. Ces auteurs ont mis en évidence que le fait que l'accroissement de la taille de l'exploitation nécessitait des ressources en travail supplémentaires et aussi impliquait plus de contraintes financières pour l'exploitant ce qui peut freiner l'adoption. Ceci par conséquent, confirme notre deuxième hypothèse.

Un des objectifs spécifiques de cet article est de déterminer le taux d'adoption de la technologie "Microdose" selon la localité. Du résultat de l'estimation, il est ressorti que la localité n'affecte pas la probabilité d'adoption de la technologie "Microdose". A ce sujet, il serait nécessaire d'apporter quelques explications y relatives.

En effet, en matière d'investissements en technologies agricoles, ces deux zones, à l'instar des autres localités du pays, ont peu évolué. Toutefois, l'analyse descriptive révèle qu'en matière de taux d'adoption, le Kouritenga présente $92,7 \%$ et le Zondoma 
$80 \%$. Ces pourcentages cachent une information capitale qui est du fait que 54\% des adoptions se sont effectuées sur des superficies inférieures à 1 hectare à cause des moyens limités des producteurs. De plus, la dynamique démographique contraint les ménages agricoles à étendre les superficies emblavées afin de compenser le besoin de consommation grandissant. L'amendement des sols par la matière organique, en particulier le compost, est pratiqué par la plupart des producteurs au Burkina Faso (Savadogo et al., 2017). Cette pratique, encouragée par les structures de recherche, apporte non seulement des éléments minéraux au sol mais aussi stimule l'activité des microorganismes du sol.

\section{Conclusion}

Cette étude vise à analyser et à déterminer les facteurs explicatifs de l'adoption de la technologie de micro dose dans deux localités agroécologiques nordsoudaniennes toutes confrontées à des contraintes similaires de changements climatiques. L'analyse a révélé que les facteurs explicatifs significatifs en matière d'adoption de la technologie "Microdose" sont : l'équipement, la formation, le nombre d'actifs, le revenu agricole et la superficie emblavée. L'adoption de la technologie "Microdose" à grande échelle pourrait se réaliser avec les agriculteurs s'engageant dans les démarches innovantes. Cependant, face à la spécificité des caractéristiques des agriculteurs et de leur contexte de production, il reste difficile de généraliser les résultats à tous les types d'innovations et dans tous les contextes. D'autres analyses ex-ante de l'adoption peuvent être conduites sur le rôle des préférences des agriculteurs, afin d'identifier d'autres facteurs d'hétérogénéité susceptibles d'orienter les politiques publiques. Les agriculteurs font face à des risques inhérents à la production agricole, mais la mise en place d'une innovation sur l'exploitation agricole implique des incertitudes supplémentaires.

\section{CONFLIT D'INTERETS}

Les auteurs déclarent qu'il n'y a aucun conflit d'intérêts.

\section{CONTRIBUTIONS DES AUTEURS}

HS a participé à la collecte et l'analyse des données, a élaboré le projet d'article ainsi que les corrections. IAL a participé à l'analyse des données, la correction et intégration des recommandations. JAY a Supervisé et a participé à correction du premier et dernier drafts. GB a participé à la lecture du manuscrit.

\section{REMERCIEMENTS}

L'équipe de chercheurs adresse ses remerciements auxautorités du développement rural des régions de l'Est et du Nord, les producteurs des provinces du Zondoma et du Kouritenga.

\section{REFERENCES}

Ajewole OC. 2010. Farmer's response to adoption of commercially available organic fertilizers in Oyo state, Nigeria. Afr. J. Agric. Res., 5(18): 2497-2503. http://www.academicjournals.org/AJAR

Ameur F, Hamamouche MF, Kuper M, Benouniche M. 2013. La domestication d'une innovation technique : la diffusion de l'irrigation au goutte-à-goutte dans deux douars au Maroc. Cah Agric., 22(4): $\quad 311-318 . \quad$ DOI: http://dx.doi.org/10.1684/agr.2013.0644

Baffoe-Asare R, Danquah, JA, AnnorFrempong F. 2013. Socioeconomic Factors Influencing Adoption of Codapec and Cocoa High-tech Technologies among Small Holder Farmers in Central Region of Ghana. American Journal of Experimental Agriculture, $\quad 3(2)$ : $\quad 277-292$. http://www.sdiarticle1.org/prh/AJEA_2/ 2013/1366794178-1-Original\%20 Manuscript.pdf

Barry S. 2016. The Socio-economic and Institutional Determinants of Adoption of improved Cowpea Varieties in Northern Burkina Faso. AJAEES, 14(2): 1-12. 
Bationo A, Ngaradoum D, Youl S, Lompo F, Fening JO. 2018. Improving the Profitability, Sustainability and Efficiency of Nutrients Through Site Specific Fertilizer Recommendations in West Africa Agro-Ecosystems. Springer, 1: 241-252. DOI https://doi.org/10.1007/ 978-3-319-58789-9

Belmekki M, Mrabet R, Gharous ME, Moussadek R, Halima OI, Mohamed M, Bencharki B. 2014. Effets du non labour et de la gestion des résidus sur la conservation de l'eau et la qualité d'un sol calcimagnésique dans les zones semiarides Marocaines. International Journal of Innovation and Applied Studies, 6(4): 792-800. http://www.ijias.issrjournals.org

Bello OB, Azeez MA, Mahmud J, Afolabi MS, Ige SA, Abdulmaliq, SY. 2012. Evaluation of grain yield and agronomic characteristics in drought-tolerant maize varieties belonging to two maturing groups. International Journal of Plant Research, 2(2): 14-21. DOI: http://10.5923/j.plant.20120202.03

Bello S, Ahanchede A, Amadji G, Gbehounou G, Aho N. 2012. Effet de la fumure minérale sur l'enherbement et la production de l'oignon (Alliumcepa L.) au Nord-Est du Bénin. Int. J. Biol. Chem. Sci., $\quad$ 6(6): 4058-4070. http://ajol.info/index.php/ijbcs.

Blazy JM, Carpentier A, Thomas A. 2011. The willingness to adopt agro-ecological innovations: Application of choice modelling to Caribbean banana planters. Ecological Economics, 72: 140-150. https://doi.org/10.1016/j.ecolecon.2011.0 9.021

Bontkes TS, Van KH. 2003. Modelling the dynamics of agricultural development at farm and regional level. Agric Syst, 76: 379-396. https://doi.org/10.1016/S0308$521 \mathrm{X}(02) 00128-2$

CILSS. 2012. Bonnes Pratiques Agro-SylvoPastorales d'Amélioration Durable de la Fertilité des Sols au Burkina Faso. Commission Européenne Délégation au Burkina Faso/Comité permanent InterEtats de Lutte contre la Sécheresse au Sahel, Ouagadougou ; 194.
Dugué P, Djamen Nana P, Faure G, Le Gal PY. 2015. Dynamiques d'adoption de l'agriculture de conservation dans les exploitations familiales : de la technique aux processus d'innovation. Cah Agric, 24: 60-68. DOI: http://10.1684/agr.2015.0748

El Hadraoui Y. 2013. Etude diachronique de l'occupation du sol et de modélisation des processus érosifs du bassin versant $\mathrm{du}$ Bouregreg (Maroc) à partir des données de l'Observation de la Terre. Mémoire d'Ingénieur GéomètreTopographe. CNAM. Maroc; 88.

Folefack PD, Sale A, Wakponou A. 2012. Facteurs affectant l'utilisation de la fumure organique dans les exploitations agricoles en zone sahélienne du Cameroun. Afrique Science, 8(2): $22-$ 33.

Gelgo B, Mshenga P, Zemedu L. 2016. Analysing the Determinants of Adoption of Organic Fertilizer by Smallholder Farmers in Shashemene District, Ethiopia. International Journal of Agricultural Economics, 4(1): 117-124. DOI: http://10.11648/j.ijae.20160104.14

Gedikoglu H, McCann L. 2007. Impact of offfarm income on adoption of conservation practices. Selected Paper at the American Agricultural Economics Association Annual Meeting, Portland, OR, $28 \mathrm{p}$.

Gedikoglu H, McCann L, Artz G. 2011. OffFarm Employment Effects on Adoption of Nutrient Management Practices. Agricultural and Resource Economics Review, 40(2): 293-306. DOI: https://doi.org/10.1017/S1068280500008 078

Gomgnimbou APK, Savadogo PW, Nianogo JA, Millogo-Rasolodimby J. 2010. Pratiques agricoles et perceptions paysannes des impacts environnementaux de la cotonculture dans la province de la Kompienga (Burkina Faso). Sciences \& Nature, 7(2): 165-175.

DOI : http://dx.doi.org/10.4314/scinat.v7i2.599 $\underline{60}$

Gueye B, Freudenberger KS. 1991. Introduction à la méthode accélérée de 
recherche participative (MARP) Rapid Rural Appraisal : quelques notes pour appuyer une formation pratique; IIED, $70 \mathrm{p}$.

Grist N, Harvey B. 2017. Encadrer l'innovation liée à la résilience climatique pour les agriculteurs' du Sahel, Edition n ${ }^{\circ}$, 22p.

Jacquot A. 2000. Les modèles Econométriques-Logit-Probit-Tobit. CNAF-Bureau des prévisions, 104p.

Kaboré WTT, Hien E, Zombré P, Coulibaly A, Houot S, Masse D. 2011. Valorisation de substrats organiques divers dans l'agriculture péri-urbaine de Ouagadougou (Burkina Faso) pour l'amendement et la fertilisation des sols: Acteurs et pratiques. Biotechnol. Agron. Soc. Environ, 15(2): 271-286. http://www.pressesagro.be/ojs/index.php /base/article/view/539/892

Kariyasa K, Dewi A. 2011. Analysis of Factors Affecting Adoption of Integrated Crop Management Farmer Field School (Icm-Ffs) in Swampy Areas. International Journal of Food and Agricultural Economics, 1(2): 29-38. https://ageconsearch.umn.edu/bitstream/ 160092/2/Vol\%201\%20No\%202\%202938.pdf

Klerkx L, Van Mierlo B, Leeuwis C. 2012. Evolution of Systems Approaches to Agricultural Innovation: Concepts, Analysis and Interventions, Darnhofer I, Gibbon D, Dedieu B (eds). Springer: Dordrecht ; 457-483.

Lavison R. 2013. Factors Influencing the Adoption of Organic Fertilizers in Vegetable Production in Accra, Msc Thesis, Accra Ghana, 131p.

Mabah Tene GL, Temple L, Havard M. 2013. Les déterminants de l'adoption d'innovations techniques sur maïs au Cameroun, une contribution à la sécurisation alimentaire. Tropicultura, 31(2):

137-142.

http://www.tropicultura.org/text/v31n2/1 37.pdf

Mariano MJ, Villano R, Fleming E. 2012. Factors influencing farmers' adoption of modern rice technologies and good management practices in the Philippines.
Agricultural Systems, 110: 41-53. https://doi.org/10.1016/j.agsy.2012.03.01 0

Mignouna B, Manyong M, Rusike J, Mutabazi S, Senkondo M. 2011. Determinants of Adopting Imazapyr-Resistant Maize Technology and its Impact on Household Income in Western Kenya: AgBioforum, New Economy Handbook, 14(3): 158163. http://agbioforum.org/v14n3/ v14n3a07-mignouna.htm

Milou E. 2012. Désertification et réponses paysannes. AGRIDAPE : Revue sur l'Agriculture Durable à Faibles Apports Externes, 23(3): 4-8.

Obisesan A. 2014. Gender Differences in Technology Adoption and Welfare Impact among Nigerian Farming Households, MPRA Paper No. 58920. http://mpra.ub.uni-muenchen.de/58920/

Okunlola O, Oludare O, Akinwalere B. 2011. Adoption of new technologies by fish farmers in Akure, Ondo state, Nigeria. Journal of Agricultural Technology, 7(6):1539-1548.

Ouédraogo M. 2010. Perception des stratégies d'adaptation aux changements des précipitations : cas des paysans du Burkina Faso. Sécheresse, 21(2): 87-96. DOI :

http://www.jle.com/10.1684/sec.2010.02 44

Ouédraogo M. 2012. Impact des changements climatiques sur les revenus agricoles au Burkina Faso. Journal of Agriculture and Environment for International Development, 106(1): 3 - 21.

Pieri C. 1992. Fertility of soils: a future for farming in the West African Savannah. Springer-Verlag : Berlin; 1-42.

Reenberg A. 1996. A hierarchical approach to land use and sustainable agricultural systems in the Sahel. Q. J. Int. Agric., 35(1): 63-77.

Rogers EM. 1995. The Diffusion of Innovation $\left(4^{\text {th }}\right.$ Edn $)$. Free Press: New York, NK.

Ruas JF, Benoît-Cattin M. 1991. Modélisation technico-démographique des futurs alimentaires du Burkina Faso. Cah. Rech. Dev, 29: 1-13.

SADAOC. 1999. Transformation du système alimentaire pour faire face aux défis 
d'une population urbaine croissante. In Actes de la Table Ronde de Bamako. Ouagadougou: Fondation SADAOC : Ouagadougou ; 62p.

Sale A, Folefack DP, Obwoyere GO, Wati NL, Lendzemo WV. Wakponou A. 2014. Changements climatiques et déterminants d'adoption de la fumure organique dans la région semi-aride de Kibwezi au Kenya. Int. J. Biol. Chem. Sci., 8(2): 680-694. DOI : http://dx.doi.org/10.4314/ijbcs.v8i2.24

Savadogo PW, Zi Y, Sanou AK, Nacro, HB, Lompo F, Sedogo MP. 2017. Effets combinés du compost, du Paraquat et de la Lambdacyhalothrine sur la macrofaune du sol sous culture pluviale de sésame (Sesamum indicum L.) au Burkina Faso. Int. J. Biol. Chem. Sci, 11: 2658-2670. DOI : https://dx.doi.org/ 10.4314/ijbcs.v11i6.9

Sauer J, Zilberman D. 2009. Innovation behaviour at farm level-Selection and identification. In $49^{\text {th }}$ Annual Meeting of the German. Association of Agricultural Economics and Sociology, GEWISOLA: Kiel; 31p.

Simtowe F. 2011. Determinants of Agricultural Technology adoption: the Case of Improved Pigeon pea Varieties in Tanzania. Quarterly Journal of International Agriculture, 50(4): 325345.

Sotamenou J. 2012. Les facteurs d'adoption du compost en agriculture urbaine et périurbaine au Cameroun, Terrains \& Travaux, 1(20): 173-187.

Uematsu H, Mishra A. 2010. Can Education Be a Barrier to Technology Adoption? Selected Paper Prepared for Presentation at the Agricultural \& Applied Economics Association (AAEA). CAES, \& WAEA Joint Annual Meeting: Denver, Colorado.

Yabi JA, Bachabi F-X, Labiyi IA, Ode CAAyena R L. 2016. Déterminants socio-économiques de l'adoption des pratiques culturales de gestion de la fertilité des sols utilisées dans la commune de Ouaké au Nord- Ouest du Bénin. Int. J. Biol. Chem. Sci., 10(2): 779-792 ;

DOI

http://dx.doi.org/10.4314/ijbcs.v10i2.27

Zakari S. 2017. Adoption des technologies et pratiques d'agriculture intelligente face au climat dans les sites ccafs - (Niger). Rapport final. Technical Report, 47p. DOI: http://10.13140/RG.2.2.25400. 75526.

Zongo B. 2016. Stratégies innovantes d'adaptation à la variabilité et au changement climatiques au Sahel : Cas de l'irrigation de complément et de l'information climatique dans les exploitations agricoles du Burkina Faso. Thèse En Cotutelle, Institut International d'Ingénierie de l'Eau et de l'Environnement (2iE), p 259. 